\title{
STATISTICAL PROPERTIES OF QSOs
}

\author{
A. BRACCESI \\ Istituto di Fisica 'A. Righi', Bologna, Italy
}

\begin{abstract}
Counts of quasi-stellar objects selected by their optical properties imply evolutionary properties similar to those found by examining the radio-selected objects. The optical luminosity functions of the 3CR and optically-selected quasi-stellar objects are compared. Data on the radio emission and optical variability of the optically-selected sample are presented.
\end{abstract}

In the past three years $C$. R. Lynds has obtained spectra of 31 quasi-stellar objects in the $13^{\mathrm{h}},+35^{\circ}$, field (Braccesi et al., 1970); 24 of these objects constitute a complete sample comprising all the QSOs of the field which have an infrared magnitude $i<17.65$. I would like to report here a preliminary discussion of this material which has been carried out by G. Grueff and myself in the usual framework of the evolutionary cosmological hypothesis, and to present some data on the radio-emission and the optical variability of the objects.

A strong cosmological evolution for the QSOs is already evident from the numbermagnitude counts.

In fact Sandage and Luyten (1969) and, independently, Braccesi and Formiggini (1969) have found slopes of 1.88 and 1.80 for the counts.

This is a very remarkable result since it depends only on the optical properties of the objects, whereas it has been pointed out (Braccesi and Formiggini, 1969; Longair and Scheuer, 1971) that Schmidt's (1968) analysis of the 3CR quasars is conclusive only in showing the evolution of the radio properties of the objects.

In order to proceed further in the comparison of the QSOs and the 3CR quasars, let us assume that all the objects have the same evolutionary properties and use the two samples together in deriving the evolution.

It has been shown by Schmidt (1968) that cosmological evolution is such a predominant phenomenon, that its derived properties depend very little on the actual choice of a cosmological model, that is to say that QSOs are a very poor tool for investigating the geometry of space-time.

Thus we shall restrict ourselves to considering the relativistic cosmological model with $q_{0}=1$ and $H=75 \mathrm{~km} \mathrm{~s}^{-1} \mathrm{Mpc}^{-1}$.

Let us now consider all the objects of the two complete samples which have $z_{\max }$, (i.e. the $z$ to which they should be moved in order to be dropped from the sample), larger than a given $z=z_{0}$. This particular selection of objects is homogeneous in $z$ up to $z=z_{0}$, thus their number as a function of $z$ is proportional to the evolved volume $V^{\prime}$ defined by:

$$
V^{\prime}(z)=\int_{0}^{z} \frac{\mathrm{d} V(z)}{\mathrm{d} z} \varrho(z) \mathrm{d} z
$$


where $V(z)$ is the observable volume up to a given $z$ in the cosmological model considered and $\varrho(z)$ represents the evolution as a variation of the cosmic density of objects as a function of $z$.

With the small number of objects at our disposal (57), for any choice of $z_{0}$, the

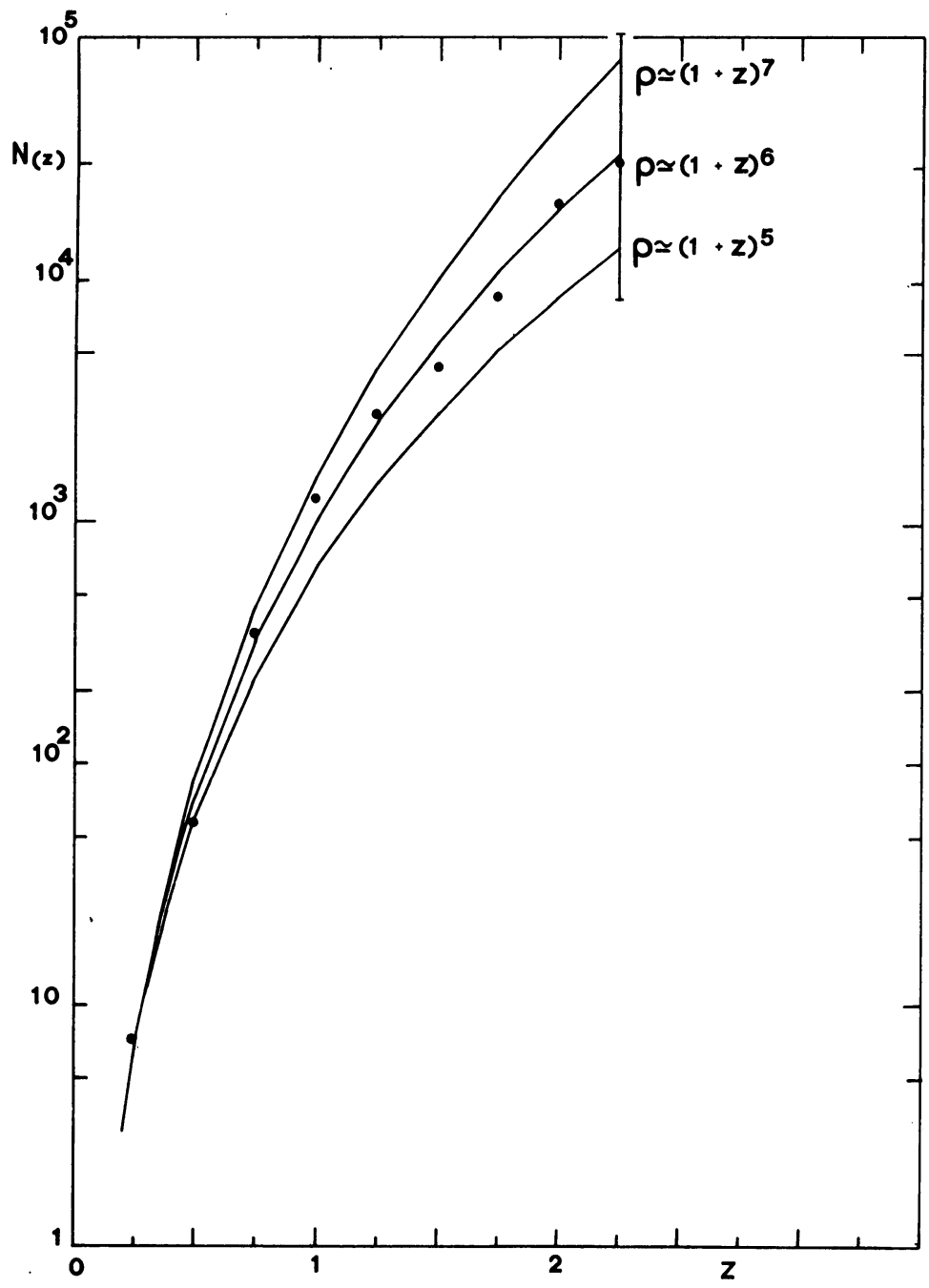

Fig. 1. The number-redshift relation of the quasi-stellar objects from the complete $3 C R$ and radio-quiet samples. These numbers are proportional to the evolved volume $V^{\prime}$.

counts cover only a very limited range in $z$. It is however possible to change $z_{0}$, and thus to cover with independent counts the range from $z=0.3$ to $z=2.25$. Then these counts are normalized one against the other and $V(z)$ is obtained over the full range 
of redshifts. The result is shown in Figure 1, where it is compared with what is expected for an evolution given by:

$$
\varrho(z)=\varrho_{0}(1+z)^{n}
$$

with $n=5,6,7$.

The curve for $n=6$ fits the data remarkably well. The uncertainty of the result has been estimated using a Monte Carlo analysis and amounts to about one power in $(1+z)$ at one rms deviation confidence level.

We can now check whether the assumption that the objects of the $3 \mathrm{CR}$ sample and of the radio-quiet sample have the same evolutionary properties was correct. To do this we can compute the average $V^{\prime} / V_{m}^{\prime}$ (the ratio of the evolved volume corresponding to the actual $z$ of an object, to the evolved volume corresponding to the $z_{\max }$ of the objects).

If the proper evolution was applied we should find $V^{\prime} / V_{m}^{\prime}=0.5$. In fact for the 3CR objects we find 0.50 and for the QSOs $0.51^{*}$.

This shows that there is no indication of a different evolution for the two samples,

\section{TABLE I}

The local luminosity function of QSOs and of 3CR QSS.

The number of objects per magnitude in a volume of $10^{77} \mathrm{~m}^{3}$ is given.

\begin{tabular}{lll}
$M_{\mathrm{B}}$ & $\begin{array}{l}Q_{0} \\
(\mathrm{QSO})\end{array}$ & $\begin{array}{l}Q_{0} \\
(3 \mathrm{CR} \text { QSS) }\end{array}$ \\
\hline$-25,-26$ & - & 0.3 \\
$-24,-25$ & 3 & 0.9 \\
$-23,-24$ & 15 & 1.1 \\
$-22,-23$ & 38 & 2.7 \\
$-21,-22$ & 1900 & 1.4 \\
$-20,-21$ & 1800 & - \\
$-19,-20$ & - & - \\
$-18,-19$ & 2500 & - \\
$-17,-18$ & 280000 & - \\
\hline
\end{tabular}

that is to say there is no evidence of a different evolution of the radio and of the optical properties.

We can now derive and compare the optical luminosity function of the QSOs and of the $3 C R$ quasars.

They are given in Table I. It is immediately evident from the table, that the effect of the radio selection, which is not too severe for the brightest objects, becomes more and more strong when one goes to fainter absolute magnitudes.

This shows that the average radio emission (or the fraction of radio-emitting objects) decreases with the absolute optical magnitude faster than the magnitude itself, in such

* The average $V / V_{m}$ (ratio of the non-evolved volumes) of the two samples are not identical. The $3 C R$ objects give 0.70 , the QSOs 0.65 . This reflects the different distributions of the redshifts of the two samples. 
a way that the radio selection becomes more and more important when one goes to objects of fainter absolute magnitude.*

The radio emission of the QSOs in the $13^{\mathrm{h}},+35^{\circ}$, field has been searched at $408 \mathrm{MHz}$ using the Bologna Cross Radio Telescope. The relevant data are summarized in

TABLE II

Summary of the $408 \mathrm{MHz}$ radio observations of the QSOs in the $13^{\mathrm{h}}$, $+35^{\circ}$, field. The objects are identified by the listing number of the photometric catalogue (Braccesi et al. 1970).

\begin{tabular}{lll}
\hline $\begin{array}{l}5 \text { detected objects } \\
6,1.7 \text { f.u. }\end{array}$ & $(S>0.2$ f.u. $)$ & \\
$66,0.7$ f.u. & $58,0.5$ f.u. & $60,0.3$ f.u. \\
\hline 170 objects not detected & $\begin{array}{l}\text { individual fluxes } \\
\text { average flux }\end{array}$ & $<0.2$ f.u. \\
& & $<0.01$ f.u.
\end{tabular}

Table II. The small number of objects detected at a flux level which is about 30 times fainter than the limit of the $3 \mathrm{CR}$, and the extremely small average flux of the objects not individually detected agree with the above mentioned interpretation.

During the past four years we have been able to monitor the optical variability of the objects using plates obtained with the Palomar 48-in. Schmidt.

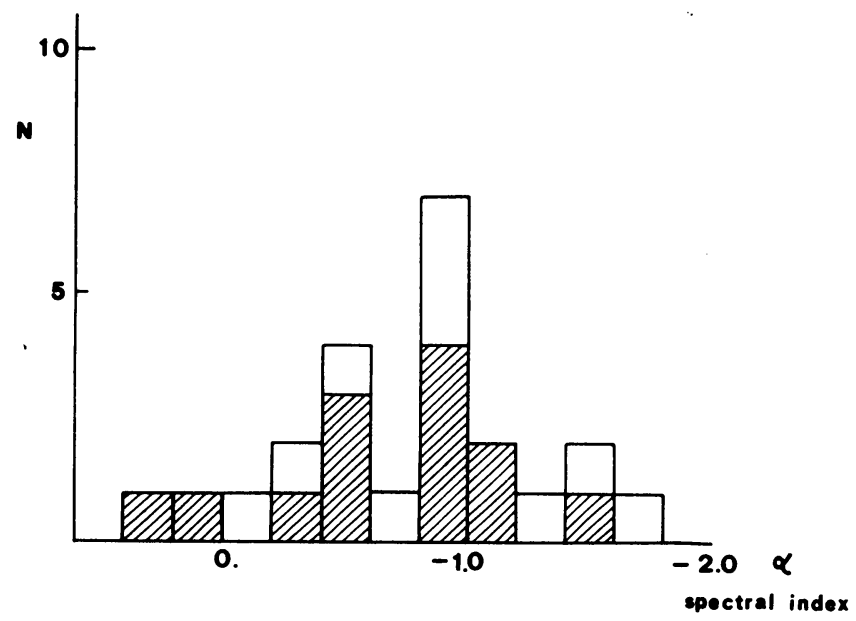

Fig. 2. The relation between the optical spectral index and the variability. The hatched area refers to the more variable objects.

All the 175 objects of the photometric catalogue (Braccesi et al., 1970) have been monitored. A large majority of them have shown a certain degree of variability, the strongest being one of the few which have been detected in the radio.

* If, as proposed by Schmidt (1970) the distributions of the ratios of the optical and radio fluxes were the same at all absolute optical magnitudes, the effect of the radio selection would not increase dramatically along the optical luminosity function in the way observed. 


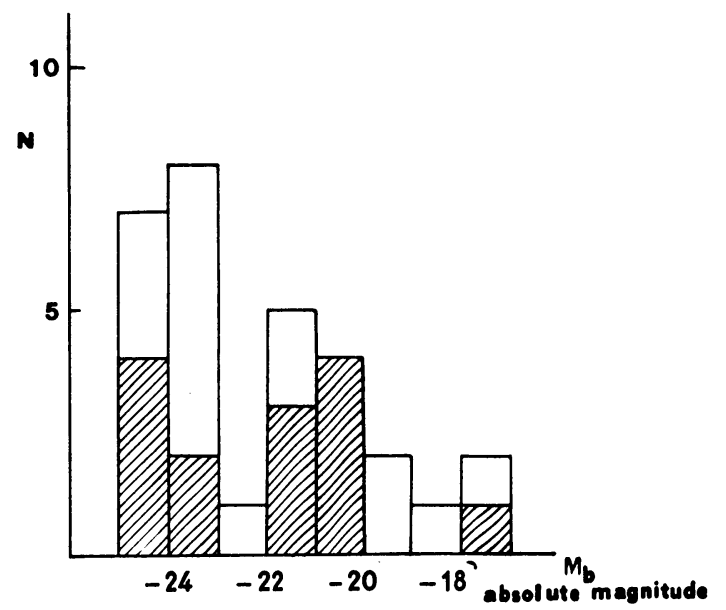

Fig. 3. The relation between the absolute optical magnitude and the variability. The hatched area refers to the more variable objects.

We have tried to correlate the amount of variability with other physical parameters: namely the absolute magnitude and the spectral index of the optical continua. It can be seen from Figures 2 and 3 that no correlation is evident.

\section{References}

Braccesi, A. and Formiggini, L.: 1969, Astron. Astrophys. 3, 364.

Braccesi, A., Formiggini, L., and Gandolfi, E.: 1970, Astron. Astrophys. 5, 264.

Longair, M. S. and Scheuer, P. A. G.: 1971, this volume, p. 470.

Sandage, A. and Luyten, W. J.: 1969, Astrophys. J. 155, 913.

Schmidt, M.: 1968, Astrophys. J. 151, 393.

Schmidt, M.: 1970, Semaine d'Étude, Pontificia Accademia delle Scienze, Roma. 